\title{
Suppression of dual-specificity phosphatase-2 by hypoxia increases chemoresistance and malignancy in human cancer cells
}

Shih-Chieh Lin, ${ }^{1}$ Chun-Wei Chien, ${ }^{1}$ Jenq-Chang Lee, ${ }^{2}$ Yi-Chun Yeh, ${ }^{1}$ Keng-Fu Hsu, ${ }^{3}$ Yen-Yu Lai, ${ }^{4}$ Shao-Chieh Lin, ${ }^{2}$ and Shaw-Jenq Tsai ${ }^{1,4}$

${ }^{1}$ Institute of Basic Medical Sciences, ${ }^{2}$ Department of Surgery, ${ }^{3}$ Department of Obstetrics and Gynecology, and

${ }^{4}$ Department of Physiology, College of Medicine, National Cheng Kung University, Tainan, Taiwan.

\begin{abstract}
Hypoxia inducible factor-1 (HIF-1) is the master transcriptional regulator of the cellular response to altered oxygen levels. HIF-1 $\alpha$ protein is elevated in most solid tumors and contributes to poor disease outcome by promoting tumor progression, metastasis, and resistance to chemotherapy. To date, the relationship between HIF-1 and these processes, particularly chemoresistance, has remained largely unexplored. Here, we show that expression of the MAPK-specific phosphatase dual-specificity phosphatase-2 (DUSP2) is markedly reduced or completely absent in many human cancers and that its level of expression inversely correlates with that of HIF-1 $\alpha$ and with cancer malignancy. Analysis of human cancer cell lines indicated that HIF-1 $\alpha$ inhibited DUSP2 transcription, which resulted in prolonged phosphorylation of ERK and, hence, increased chemoresistance. Knockdown of DUSP2 increased drug resistance under normoxia, while forced expression of DUSP2 abolished hypoxia-induced chemoresistance. Further, reexpression of DUSP2 during cancer progression caused tumor regression and markedly increased drug sensitivity in mice xenografted with human tumor cell lines. Furthermore, a variety of genes involved in drug response, angiogenesis, cell survival, and apoptosis were found to be downregulated by DUSP2. Our results demonstrate that DUSP2 is a key downstream regulator of HIF-1-mediated tumor progression and chemoresistance. DUSP2 therefore may represent a novel drug target of particular relevance in tumors resistant to conventional chemotherapy.
\end{abstract}

\section{Introduction}

Hypoxia inducible factor-1 (HIF-1) is a transcription factor that functions as a master regulator of oxygen homeostasis. The HIF heterodimer comprises a constitutive $\beta$-subunit and an $\alpha$-subunit whose protein level depends on ambient oxygen content. In normoxic conditions, prolyl hydroxylase modifies two proline residues (prolines 402 and 564) within the oxygen-dependent degradation domain of HIF-1 $\alpha$ (1). This modification promotes ubiquitination and proteasomal degradation of HIF- $1 \alpha(2,3)$. Under hypoxic conditions, HIF- $1 \alpha$ escapes degradation and is free to dimerize with HIF-1 $\beta$. Dimers subsequently translocate to the nucleus and regulate transcription of a suite of hypoxia-dependent genes.

HIF- $1 \alpha$ protein levels are elevated in most solid tumors due to hypoxic stress or aberrant activation of some oncogenes (4). Clinical investigation revealed that elevation of HIF- $1 \alpha$ makes tumor cells more resistant to chemotherapy and increases the likelihood of metastasis and poor outcome (5). However, the underlying mechanism of HIF-induced chemoresistance is poorly understood.

Dual-specificity phosphatases are negative regulators of MAPKs that function by dephosphorylating both phosphotyrosine and phosphoserine/threonine residues (6). The human genome contains 30 putative DUSP genes, 11 of which are recognized as bona fide DUSPs based on the presence of an N-terminal MAPK-binding domain (7). DUSPs can be divided into 3 groups using subcellular

Conflict of interest: The authors have declared that no conflict of interest exists. Citation for this article: J Clin Invest. 2011;121(5):1905-1916. doi:10.1172/JCI44362. localization as a classification criterion (8). Class I DUSPs (DUSP1, $-2,-4$, and -5 ) are localized to the nucleus, while class II DUSPs (DUSP6, -7, and -16) reside in cytoplasm. Class III DUSPs (DUSP8, DUSP9, and DUSP10) can dephosphorylate substrates in either the nucleus or cytoplasm (8). In general, the substrates for class I and II DUSPs include ERK, p38 MAPK, and JNK, while class III DUSPs dephosphorylate only p38 MAPK and JNK (6). Their high substrate specificity and defined spatial expression make DUSPs ideal molecules to differentially regulate the complex MAPK pathways.

DUSP2, originally named phosphatase of activated cells 1 (PAC-1), was first cloned from phytohemagglutinin-stimulated human peripheral T cells as an immediate early gene (9). A nucleus-specific phosphatase, DUSP2 functions predominantly to inactivate ERK but, to a lesser degree, also inactivates p38 $\operatorname{MAPK}(9,10)$. Since DUSP2 was originally cloned from human T cells, most study to date has focused on the role of DUSP2 in immune regulation. DUSP2 mRNA levels increase in activated leukocytes, and this elevated expression is associated with inflammation (11). Mice lacking DUSP2 develop and age normally but show a reduced inflammatory response in an autoimmune model of rheumatoid arthritis (12). DUSP2 has also been found to regulate p53- and E2F1-regulated apoptosis $(13,14)$. Aside from its regulation of inflammation and cell cycle, little is known about the function of DUSP2 in other physiological and pathological processes.

Here, we report that DUSP2 mRNA and protein are markedly reduced or completely absent in many cancers. Reduced DUSP2 expression is mediated by HIF- $1 \alpha$-dependent transcriptional 
repression. Inhibition of DUSP2 expression by HIF-1 $\alpha$ led to prolonged ERK phosphorylation and increased drug resistance. In contrast, forced expression of DUSP2 induced apoptosis and abolished hypoxia-induced drug resistance. Reexpression of DUSP2 in xenografted mouse models of cancer increased drug sensitivity and caused tumor regression. In these models, DUSP2 negatively regulated many genes involved in cancer cell survival and malignancy. These findings establish DUSP2 as a critical link between hypoxia and cancer progression, malignancy, and chemoresistance. DUSP2 thus represents a promising target for novel molecular therapeutics, particularly in cancers resistant to traditional chemotherapy.

\section{Results}

DUSP2 expression is downregulated in many cancers. To investigate the functional role of DUSP2 in tumor progression, we employed quantitative RT-PCR (RT-qPCR) to analyze its expression in clinical cancer samples. Specifically, we quantified DUSP2 transcript in a panel derived from normal and cancerous breast, colon, lung, ovary, kidney, prostate, liver, and thyroid (mRNA from 3 normal and 4-8 cancer samples for each organ). DUSP2 mRNA decreased versus normal in all cancerous tissues except those of the liver and thyroid (Figure 1A). To obtain a more comprehensive picture, we examined 18 sets of cervical cancer biopsy samples and 102 pairs of normal colon and colon cancer samples by immunohistochemical staining. While DUSP2 staining was evident in virtually all normal cervical epithelial cells (Figure 1B), it was markedly reduced and, in most cases (77\%), undetectable in cervical cancer cells (Figure 1, $\mathrm{B}$ and $\mathrm{C}$ ). In contrast, CAIX, a protein marker of hypoxic cells, was detected in cancer samples but not normal cervical epithelial cells (Figure 1B). In the normal colon, DUSP2 protein was detected in $91.6 \%$ of cells, while in colon cancer samples, $39.2 \%$ of cells were DUSP2-negative (Figure 1, D and E). In a majority of cancer cell samples, especially in late-stage tumors, DUSP2 levels were lower than those in adjacent normal cells (Figure 1F). Again, the level of DUSP2 was inversely correlated with the level of CAIX (Figure 1D). Further analysis of DUSP2 expression in colon cancer samples revealed that the levels of DUSP2 were inversely correlated with tumor status, nodal status, and stage of the disease (Supplemental Table 1; supplemental material available online with this article; doi:10.1172/JCI44362DS1). Taken together, these data demonstrate that expression of DUSP2 is markedly decreased in most solid cancers and that suppression of DUSP2 is associated with tumorigenesis and malignancy.

Expression of DUSP2 in cancer cells is inbibited by HIF-1 $\alpha$. To investigate the mechanism underlying loss of DUSP2 expression in cancer cells, we first evaluated DNA methylation in the DUSP2 promoter region. We determined that the DUSP2 promoter was not hypermethylated in any of the cancers assessed (data not shown) and therefore that another mechanism contributed to diminished expression. The inverse correlation of DUSP2 and CAIX staining in colon and cervical cancers led us to hypothesize that DUSP2 expression may be downregulated by hypoxia. To test this hypothesis, we exposed several cancer cell lines - including HeLa (cervical cancer), MDA-MB231 (breast cancer), MDA-MB468 (breast cancer), SW480 (colon cancer), HCT116 (colon cancer), Colo320DM (colon cancer), A549 (lung cancer), Ovcar3 (ovarian cancer), PC3 (prostate cancer), RL-95-2 (endometrial cancer), Hep3B (liver cancer), PLC (liver cancer), and Huh7 (liver cancer) - to hypoxic conditions for 24 hours and quantified DUSP 2 mRNA by RT-qPCR. Indeed, DUSP2 mRNA levels were markedly reduced by hypoxia in all except the 3 liver cancer cell lines (Figure 2A and Supplemental Figure 1). mRNA encoding pyruvate dehydrogenase kinase-1 (PDK1), a known hypoxia-upregulated gene (15-17), was markedly increased by hypoxia (Supplemental Figure 2), indicating that the observed DUSP2 suppression is a specifically regulated phenomenon. Importantly, a hypoxia-mediated reduction in DUSP2 protein levels paralleled the observed mRNA decline (Figure 2B). Taken together, these data demonstrate that reduced expression of DUSP2 in cancer is likely due to the inhibitory effect of hypoxia.

Next, desferrioxamine (DFO) and dimethyloxalylglycine (DMOG), compounds that cause HIF- $1 \alpha$ and HIF- $2 \alpha$ accumulation under normoxia, were used to determine whether inhibition of DUSP2 expression by hypoxia was mediated by upregulation of HIF. Treatment of multiple cancer cell lines with DFO and DMOG indeed caused a marked decrease in DUSP2 mRNA (Figure 2C), suggesting that hypoxia inhibition of DUSP2 is HIF dependent.

HeLa cells provided a tractable model system to further explore the mechanism by which HIF inhibits DUSP2 expression. To probe this question, we first used siRNA to knock down HIF- $1 \alpha$ and HIF-2 $\alpha$ (Figure 2D). This knockdown experiment demonstrated that inhibition of DUSP2 was primarily controlled by HIF-1 $\alpha$, whereas HIF- $2 \alpha$ played a minor role (Figure 2, D-F). To further confirm this finding, we transfected HeLa cells with either wildtype or oxygen-insensitive HIF-1 $\alpha$ (P402A/P564A) cDNA but left them under normoxia. While wild-type HIF-1 $\alpha$ partially inhibited DUSP2 expression, oxygen-insensitive HIF-1 $\alpha$ (P402A/P564A) completely ablated it, demonstrating that HIF- $1 \alpha$ activity is sufficient to inhibit DUSP2 expression (Figure 2G).

To search for the potential HIF- $1 \alpha$-binding site, we employed a bioinformatics platform (the Binding Element Searching Tool [The Best], http://www.binfo.ncku.edu.tw/thebest/) to analyze the promoter region of the human DUSP2 gene. A putative hypoxia response element (HRE) in the promoter of the human DUSP2 gene was predicted (Figure $2 \mathrm{H}$ ). Multiple sequence alignment demonstrated that the DUSP2 HRE is highly conserved among mammalian species (Supplemental Figure 3), suggesting evolutionary importance. Indeed, promoter activity assays demonstrated that true hypoxia and chemical-mimicked hypoxia (DFO treatment) inhibited transcription of the wild-type human DUSP2 gene but had no effect on HRE-mutated promoters (Figure $2 \mathrm{H}$ ). Furthermore, overexpression of oxygen-insensitive HIF- $1 \alpha$ inhibited the DUSP2 promoter under normoxic conditions in the responsive HeLa cells but not in the unresponsive Hep3B cells (Figure 2I). Finally, chromatin immunoprecipitation-PCR results showed that HIF- $1 \alpha$ specifically binds to the predicted HRE under hypoxic conditions (Figure 2J). Taken together, these data demonstrate that hypoxia-inhibited DUSP2 expression is primarily controlled by HIF-1 $\alpha$ and that this regulation is common to multiple cancer cell types.

Hypoxic inhibition of DUSP2 expression causes prolonged ERK phosphorylation. To investigate the biological consequences of DUSP2 inhibition by HIF-1, we placed HeLa in hypoxic conditions and assessed MAPK phosphorylation. Western blotting showed that phosphorylation of ERK was markedly increased in cells exposed to hypoxia (Figure 3A), while levels of phosphorylated p38 MAPK and JNK were unchanged (Supplemental Figure 4). Prolonged ERK phosphorylation was also seen with DFO/DMOG treatment (Figure 3B) and HIF-1 $\alpha$ overexpression (Figure 3C). Confocal microscopic analysis revealed that, under hypoxic conditions, phosphorylated ERK accumulated in the nucleus (Figure 3D). This distribution was well coordinated with downregulation of DUSP2, 
A

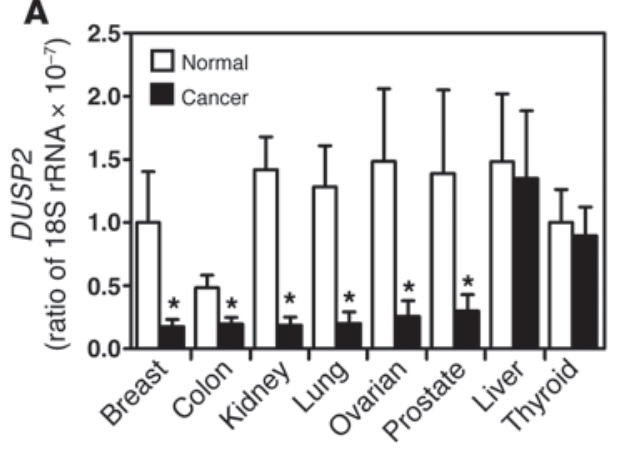

B
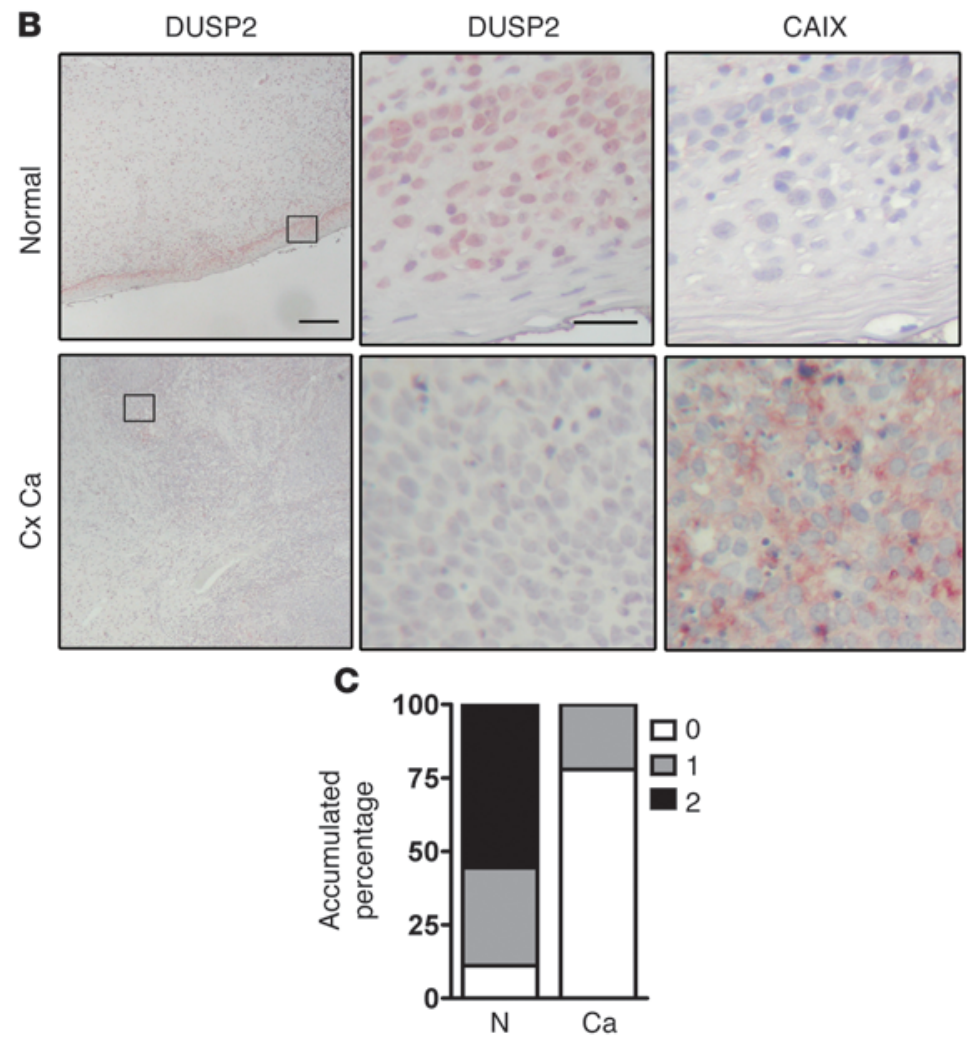

D
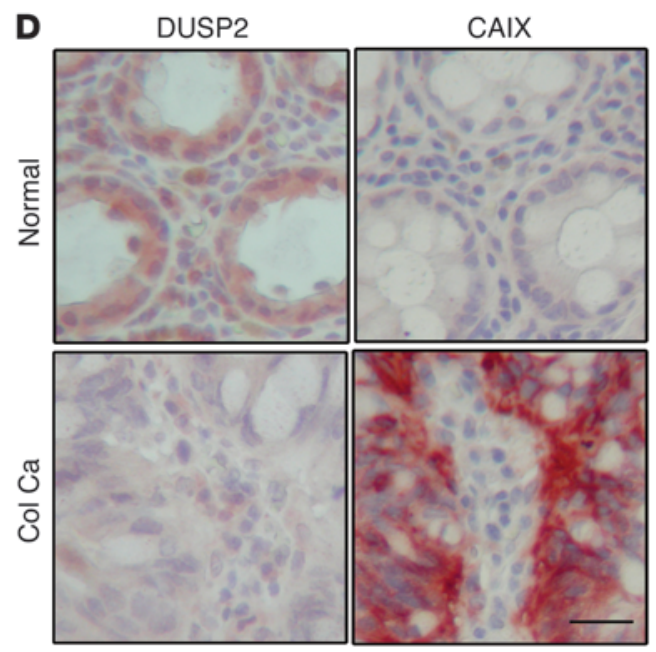

E

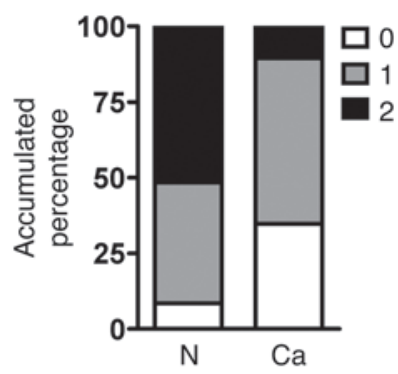

$\mathbf{F}$

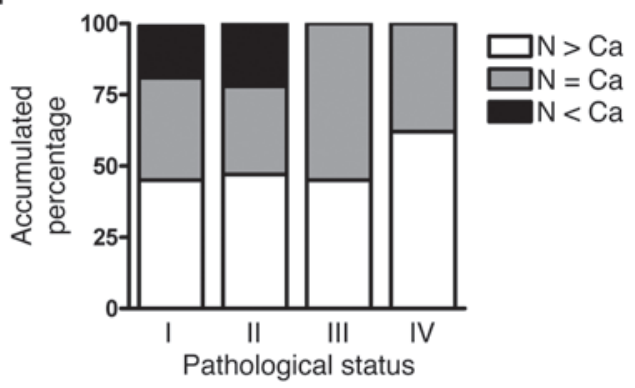

Figure 1

DUSP2 is downregulated in most cancer cells. (A) Levels of DUSP2 mRNA quantified by RT-qPCR in cDNA panels of several cancer tissues and their normal counterparts. ${ }^{*} P<0.05$ compared with normal. (B) Representative immunohistochemical staining for DUSP2 (left panels; and at higher magnification in middle panels) and CAIX (right panels) in paired normal and cancerous cervical (Cx Ca) tissues. Scale bars: $200 \mu \mathrm{m}$ and $25 \mu \mathrm{m}$ for low and high magnification, respectively. (C) Accumulated percentage of DUSP2 staining intensity in normal (N) and cancerous (Ca) cervical tissues. (D) Representative immunohistochemical staining for DUSP2 and CAIX in paired normal and colon cancer (Col Ca) tissues. Scale bars: $25 \mu \mathrm{m}$. (E) Accumulated percentage of DUSP2 staining intensity in normal and cancerous colorectal (colon) tissues. (F) Accumulated percentage of DUSP2 staining intensity in paired normal and cancerous tissues grouped by pathological stages. $\mathrm{N}>\mathrm{Ca}$, expression level is greater in normal tissue than in cancer; $\mathrm{N}=\mathrm{Ca}$, expression level is equal in normal tissue and cancer; $\mathrm{N}<\mathrm{Ca}$, expression level is lower in normal tissue than in cancer.

a nuclear MAPK-specific phosphatase. Time course experiments demonstrated that DUSP2 was downregulated by 4 hours after initiation of hypoxia, preceding the time at which phosphorylation of ERK increases (Figure 3, E and F). In contrast, mRNA levels of other nuclear DUSPs, DUSP1, DUSP4, and DUSP5, were upregulated by hypoxia (Figure 3E and Supplemental Figure 5). These data suggest that downregulation of DUSP2, but not other nuclear DUSPs, may specifically enable prolonged ERK phosphorylation in the setting of hypoxia.
To further confirm that this prolonged ERK phosphorylation was mediated by downregulation of DUSP2, we transiently transfected plasmids carrying cDNA for a human DUSP2-GFP fusion protein (DUSP2-GFP) or GFP alone into HeLa cells. In keeping with the hypothesis, forced expression of DUSP2-GFP but not GFP alone abolished hypoxia-induced ERK phosphorylation (Figure 3, G and $\mathrm{H}$ ). Taken together, these data demonstrate that under hypoxic conditions, HIF-1 suppresses DUSP2 expression, thus enabling prolonged ERK phosphorylation. 
A

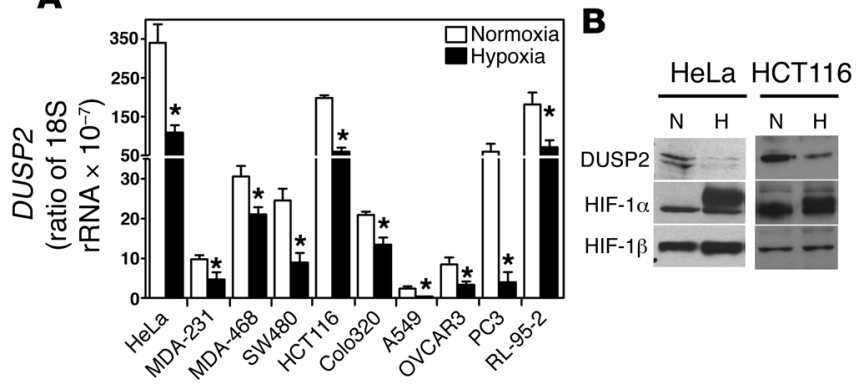

E
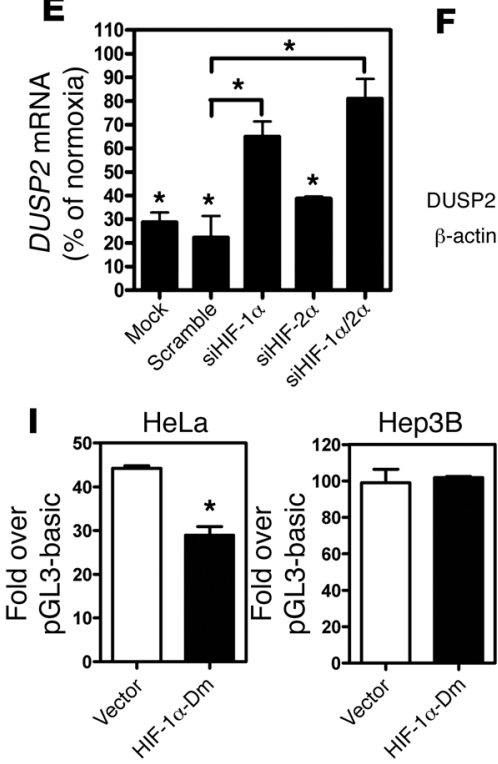

F

$\mathbf{G}$
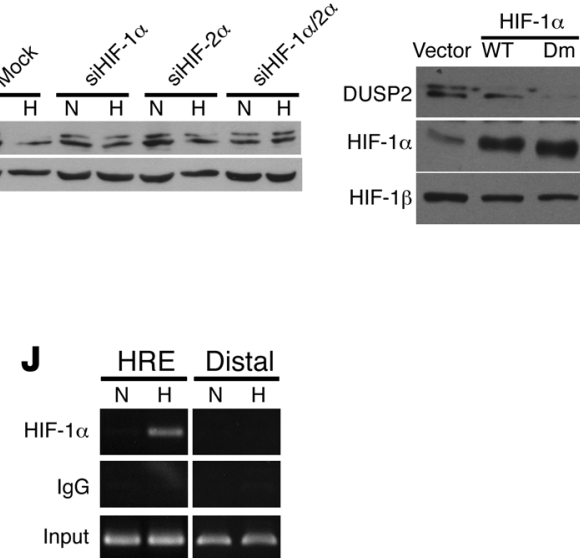
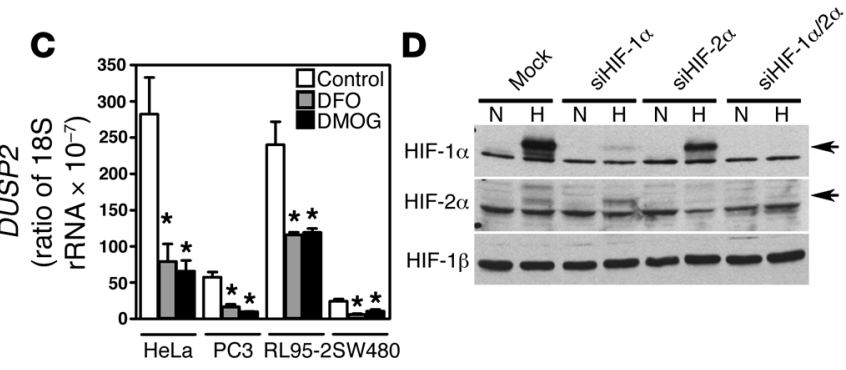

H
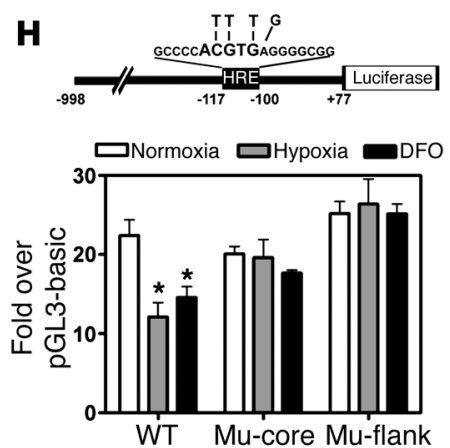

\section{Figure 2}

DUSP2 is inhibited by HIF-1 $\alpha$. (A) Cancer cell lines were exposed to normoxia and hypoxia for 24 hours, and levels of DUSP2 mRNA were quantified by RT-qPCR. (B) Representative Western blots of cells cultured under normoxia or hypoxia (H) for 24 hours showing levels of DUSP2, HIF-1 $\alpha$, and HIF-1 $\beta$. (C) Levels of DUSP2 mRNA in cancer cells treated with DFO (10 mM), DMOG (1 mM), or vehicle (Control) for 24 hours. (D) Representative Western blots of HeLa cells with HIF-1 $\alpha$ or HIF-2 $\alpha$ knockdown showing levels of HIF proteins (indicated by arrows). (E and F) Levels of DUSP2 mRNA and protein in HIF-1 $\alpha$ or HIF-2 $\alpha$ knockdown cells are shown. (G) Representative Western blots of HeLa cells transiently transfected with wild-type HIF-1 $\alpha$, P402A/P564A double-mutated HIF-1 $\alpha$ (Dm), or empty vector (pcDNA6.0) under normoxia showing the levels of DUSP2, HIF-1 $\alpha$, and HIF-1 $\beta$. (H) Schematic drawing of human DUSP2 promoter (upper panel). Core HRE is indicated in bold. Mutated bases are shown above. Promoter activity of HeLa cells transiently transfected with reporter constructs as indicated and cultured under normoxia, normoxia treated with DFO, or hypoxia for 24 hours (lower panel). Mu-core, core HRE mutated construct; Mu-flank, sequence flanking core HRE mutated construct. (I) Promoter activity of HeLa and Hep3B cells transiently transfected with reporter constructs as indicated and cultured under normoxia for 24 hours with or without overexpression of HIF-1 $\alpha-D m$. (J) ChIP-PCR results of HeLa cells exposed to normoxia or hypoxia for 4 hours. HRE, primers amplified flanking HRE region; Distal, primers amplified a fragment of 2,000 bp upstream of HRE. ${ }^{*} P<0.05$.

DUSP2 induces apoptosis and inbibits tumor growth. Given that DUSP2 was downregulated in most tumors, we then tested whether reexpression of DUSP2 in hypoxic tumors would abolish ERK-dependent cell survival and tumor growth. First, we established stable clones that carried a tetracycline-inducible DUSP2GFP fusion gene and used them to investigate functional roles of DUSP2 in cancer progression. The addition of doxycycline to the culture medium of HeLa cells carrying the inducible DUSP2-GFP gene, but not cells with GFP alone, blocked ERK phosphorylation (Figure 4A) and increased apoptosis (Figure 4B). Several other lines of stable clones were generated, and the results were similar (Supplemental Figure 6).

We then tested the functional role of DUSP2 in the growth of tumor xenografts by inoculating SCID mice with HeLa cells that carried either the inducible DUSP2-GFP fusion gene or GFP alone. Once tumor volumes reached $50 \mathrm{~mm}^{3}$, mice $(n=5$ /group) received doxycycline via drinking water to activate the transgenes. Immunohistochemical staining showed that the addition of doxycycline effectively induced the expression of GFP (GFP alone group) and DUSP2 (DUSP2-GFP group). The localization of the DUSP2GFP fusion protein was, as expected, in the nucleus, while the GFP protein was detected in cytoplasm (Supplemental Figure 7). Tumors in DUSP2-GFP mice receiving regular drinking water continued to grow, while those in DUSP2-GFP mice receiving doxycycline stopped growing and started to regress 3 days after the addition of doxycycline (Figure 4, C and D, for clone 8, and Supplemental Figure 8 for clone 13). TUNEL staining demonstrated that the number of apoptotic cells was markedly increased in doxycycline-induced DUSP2-GFP tumors (Figure 4E). In contrast, tumors in mice inoculated with cells carrying GFP alone contin- 

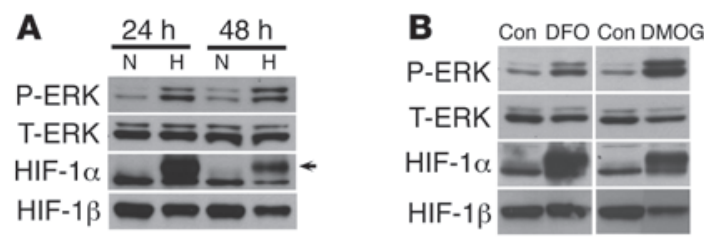
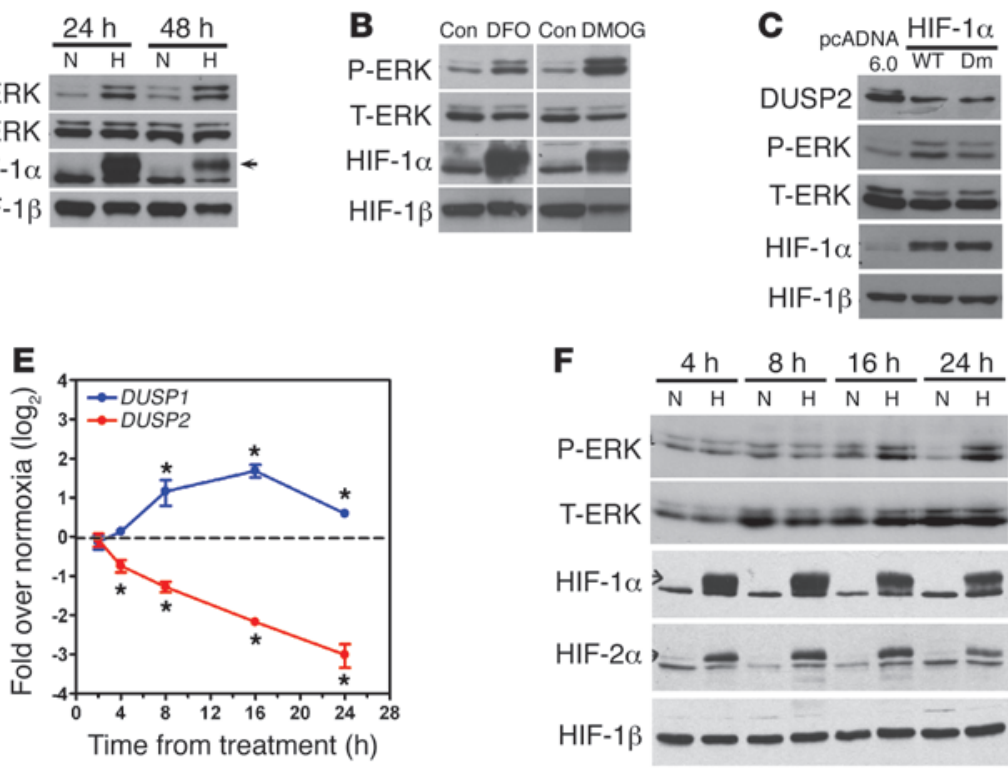

G

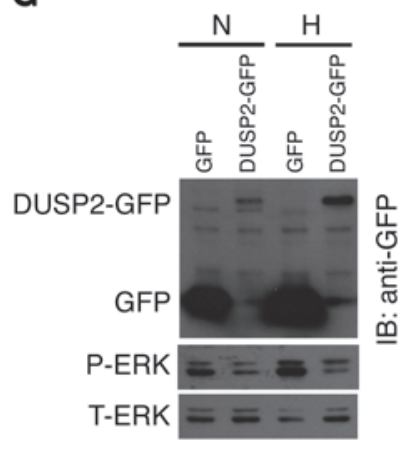

$\mathbf{F}$
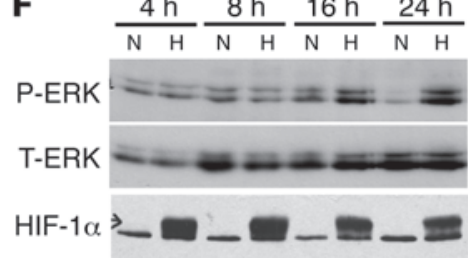

$\mathrm{HIF}-2 \alpha,-=-=-=$

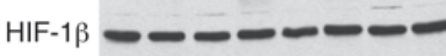

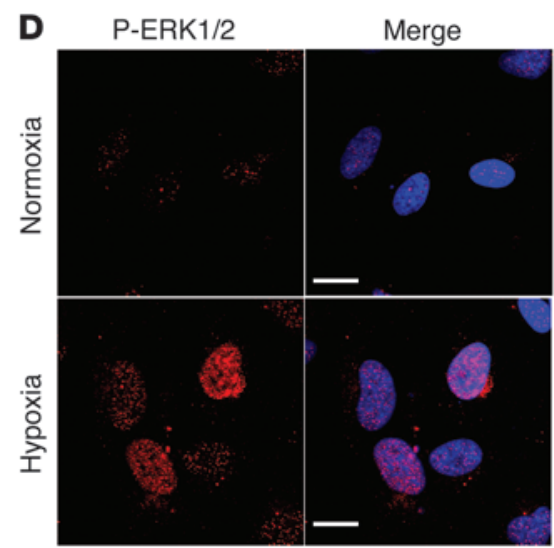

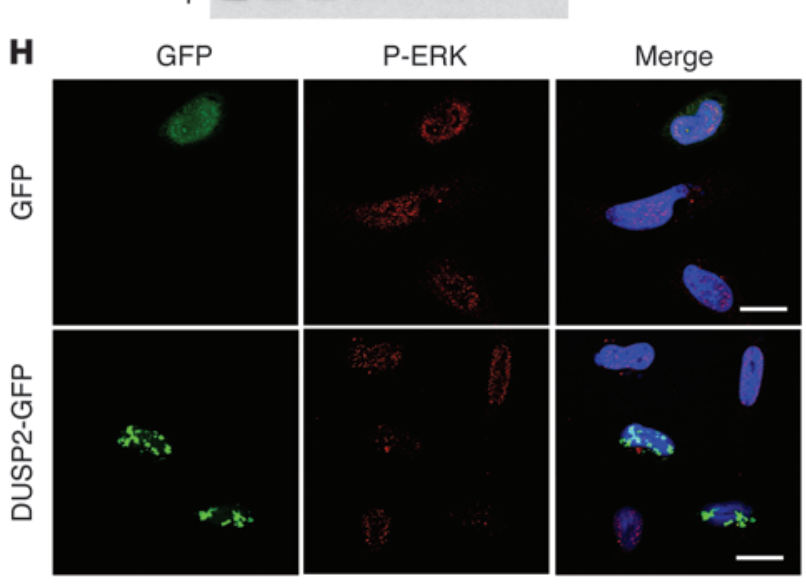

\section{Figure 3}

Suppression of DUSP2 by hypoxia leads to prolonged ERK phosphorylation. (A) Representative Western blots of HeLa cells cultured under normoxia or hypoxia for 24 or 48 hours showing levels of phosphorylated ERK (P-ERK), total ERK (T-ERK), HIF-1 $\alpha$, and HIF-1 $\beta$. Arrow indicates HIF-1 $\alpha$. (B) Representative Western blots of HeLa cells treated with DFO, DMOG, or vehicle (Con) showing levels of phosphorylated ERK (P-ERK), total ERK (T-ERK), HIF-1 $\alpha$, and HIF-1 $\beta$. (C) Representative Western blots of HeLa cells transiently transfected with wild-type HIF-1 $\alpha$, HIF-1 $\alpha-D m$, or empty vector (pcDNA6.0) under normoxia showing the levels of DUSP2, phosphorylated ERK, total ERK, HIF-1 $\alpha$, and HIF-1 $\beta$. (D) Confocal photomicrographs showing HeLa cells stained for P-ERK under normoxia and hypoxia (left panels) and merged with images from DAPI staining (Merge). Scale bars: $20 \mu \mathrm{m}$. (E) Amounts of mRNA encoding for DUSP1 and -2 in HeLa cells exposed to normoxia or hypoxia for various times. ${ }^{*} P<0.05$ compared with normoxia group (dashed line). (F) Representative Western blots of HeLa cells exposed to normoxia or hypoxia for various times showing levels of phosphorylated ERK, total ERK, HIF-1 $\alpha, \mathrm{HIF}-2 \alpha$, and HIF-1 $\beta$. (G) Representative Western blots of HeLa cells transiently transfected with GFP alone or DUSP2-GFP fusion gene cultured under normoxia or hypoxia showing levels of GFP, DUSP2-GFP, phosphorylated ERK, and total ERK. (H) Confocal photomicrographs showing green fluorescence and P-ERK staining, and merged with images from DAPI staining (Merge) in HeLa cells transfected with the DUSP2-GFP fusion gene or GFP alone. Scale bars: $20 \mu \mathrm{m}$.

ued to grow with or without doxycycline treatment (Figure 4, F and G). The wet weights of tumors in mice inoculated with DUSP2-GFP cells were significantly lower following doxycycline treatment (Figure 4D), while tumor weight in mice inoculated with cells carrying GFP alone was not different in doxycyclinetreated and control groups (Figure 4G).

We next employed loss-of-function studies to determine whether DUSP2 negatively regulates cell proliferation. Specifically, we established stable HeLa cell clones in which DUSP2 was knocked down by shRNA (Figure 5A). We generated two shRNA constructs (shDUSP2-1 and shDUSP2-2) and randomly selected clones expressing these species. The 3 clones generated from shDUSP2-1 demonstrated marked DUSP2 knockdown, while the 4 clones generated from shDUSP2-2 were not as effective (Figure 5A). Therefore, we chose two stable clones (nos. 8 and 12) from the shDUSP2-1 knockdown lines for subsequent experiments. The knockdown effect was specific, as DUSP1, DUSP4, and DUSP5 mRNAs were unaffected by the presence of shRNA (Figure 5B). DUSP2-knockdown and control (shLuciferase) HeLa cells were then inoculated into SCID mice ( $n=8$ /group). As shown in Figure 5C, tumors derived from DUSP2-knockdown cells grew significantly faster than those derived from control cells, eventually reaching at least 5 
A

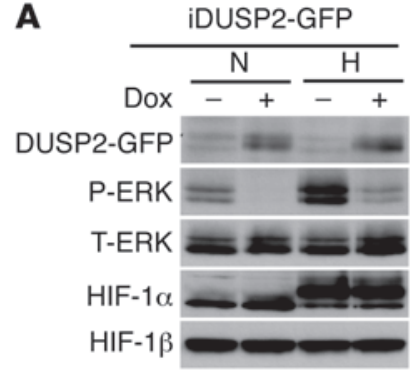

iGFP

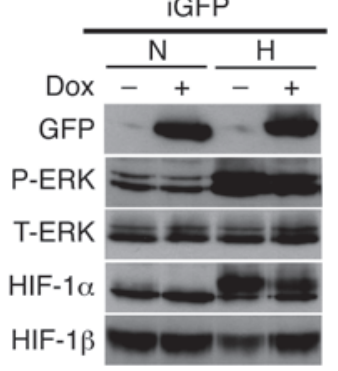

B

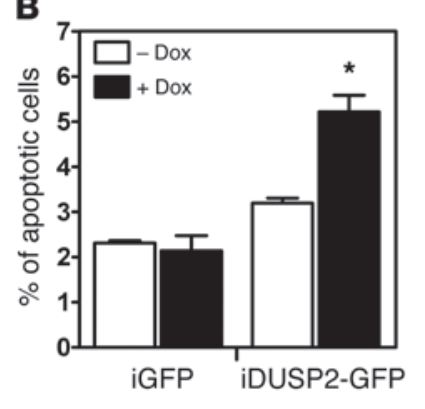

C

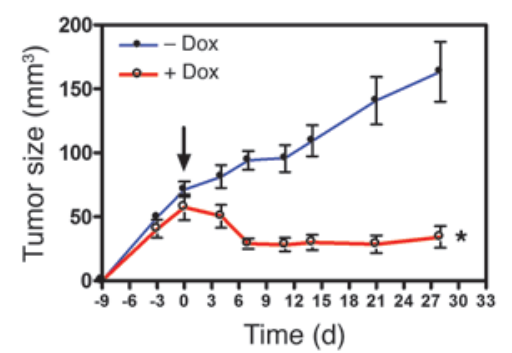

D

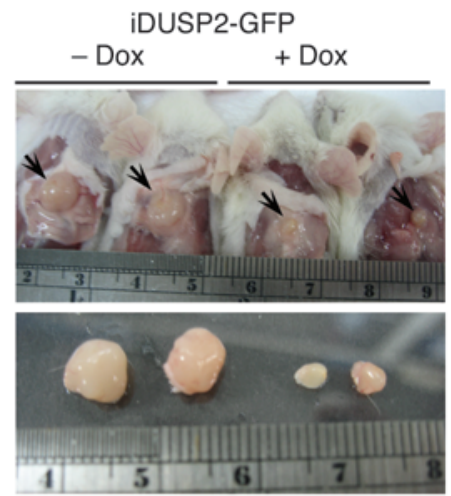

$\mathbf{F}$

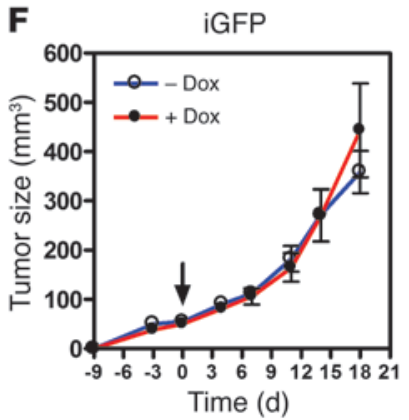

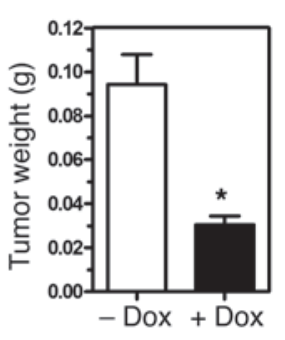

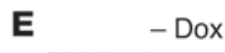

+ Dox

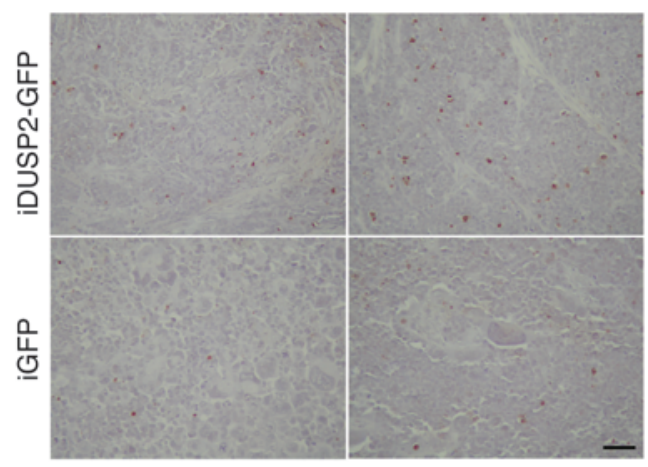

G
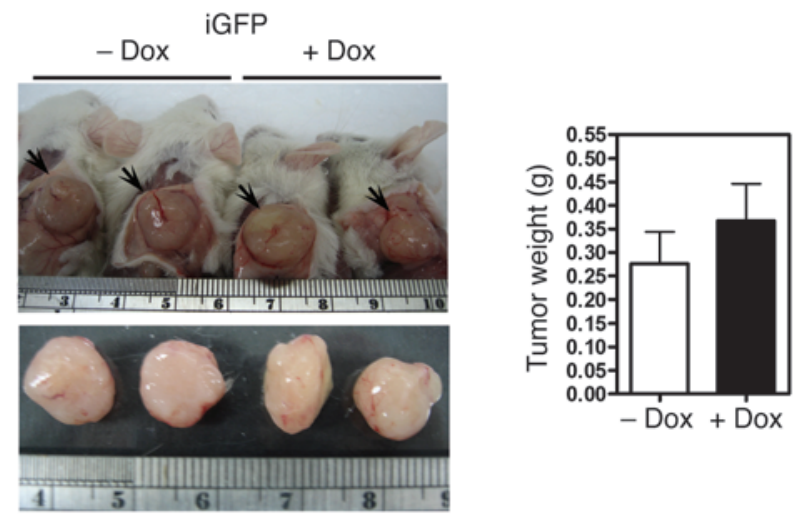

Figure 4

Reexpression of DUSP2 in cancer cells induces apoptosis and tumor regression. (A) Representative Western blots showing levels of DUSP2GFP, GFP, phosphorylated ERK, total ERK, HIF-1 $\alpha$, and HIF-1 $\beta$ in HeLa cells carrying the doxycycline-inducible DUSP2-GFP fusion gene (iDUSP2-GFP) or GFP alone (iGFP) cultured under normoxia or hypoxia in the presence (+) or (-) absence of doxycycline (Dox; $2 \mu \mathrm{g} / \mathrm{ml})$ for 24 hours. (B) Induction of apoptosis in HeLa cells carrying the doxycycline-inducible DUSP2-GFP fusion gene or GFP alone cultured under normoxia or hypoxia in the presence or absence of doxycycline $(2 \mu \mathrm{g} / \mathrm{ml})$ for 24 hours. ${ }^{*} P<0.05$ compared with group without doxycycline. (C) Growth curves of tumors with inducible DUSP2-GFP. Arrow indicates the day of addition of doxycycline. (D) Representative pictures showing tumors grown in mice ( $n=5 /$ group) inoculated with HeLa cells carrying inducible DUSP2-GFP genes without or with doxycycline treatment (left panel). Tumors are shown in situ (upper panel) and after removal (lower panel). Ruler shows centimeters. The average wet weights of tumors with inducible DUSP2-GFP on day 28 are shown in the right panel. (E) Representative pictures showing apoptotic cells (with brown nuclei) in tissue sections of xenografted tumors. Scale bar: $50 \mu \mathrm{m}$. (F and $\mathbf{G}$ ) Growth curves, pictures of tumors, and average wet weights of tumors with inducible GFP. See legends to $\mathbf{C}$ and $\mathbf{D}$. ${ }^{\star} P<0.05$ compared with groups without doxycycline treatment.

times their volume (Figure 5C and Supplemental Figure 9). Taken together, our data demonstrate that hypoxia-mediated suppression of DUSP2 is a critical factor in promoting tumor growth.

Suppression of DUSP2 contributes to bypoxia-induced drug resistance. Since hypoxic cancer cells are known to be more resistant to chemotherapy, we sought to investigate whether downregulation of DUSP2 contributes to hypoxia-mediated drug resistance. To do so, we treated HeLa and HCT116 cells with cisplatin, oxaliplatin, or paclitaxel for 24 hours under normoxia or hypoxia. While treatment with these drugs induced dose-dependent cell death under normoxic conditions (Supplemental Figure 10), the toxic effect was significantly attenuated with hypoxia (Figure 6A). DFO treatment of the same cells recapitulated the effect of hypoxia (Supplemental Figure 11), suggesting this increased drug resistance is HIF-1 $\alpha$ dependent.

To further confirm that loss of DUSP2 function contributes to increased drug resistance, we treated DUSP2-knockdown cells with 
A

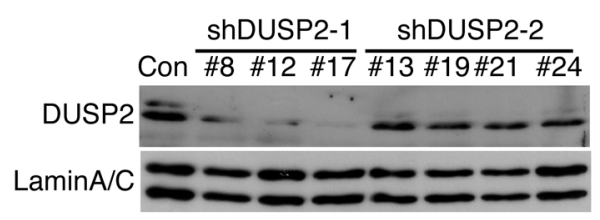

B
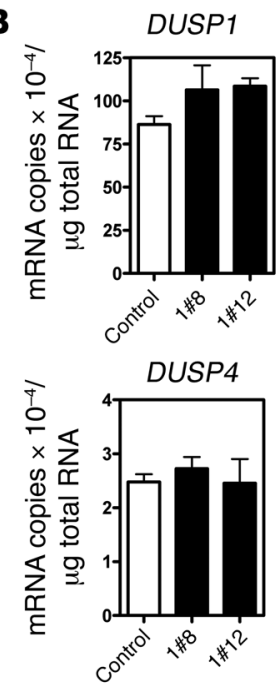

DUSP2

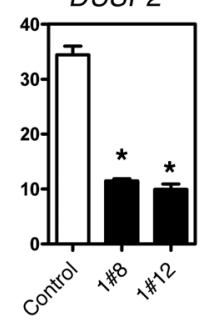

DUSP5

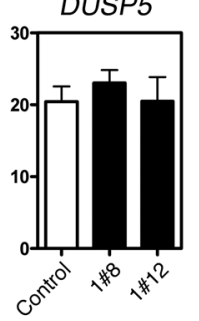

C

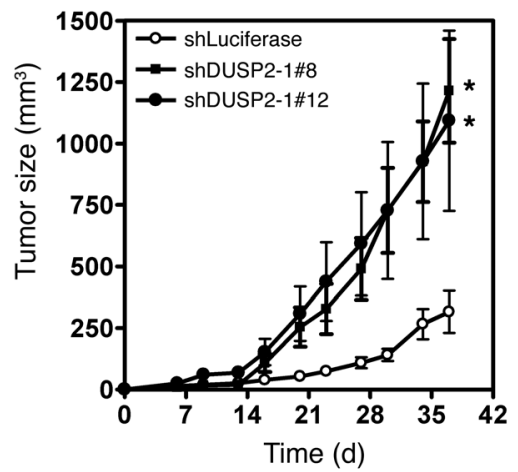

\section{Figure 5}

Loss of function of DUSP2 promotes tumor growth. (A) Representative Western blots of several stable clones of HeLa cells transfected with shRNA against human DUSP2 showing levels of DUSP2 and lamin A/C. Con, HeLa cells transfected with shRNA against luciferase; shDUSP2-1 and shDUSP2-2 represent two shRNAs targeting different areas of DUSP2. Numbers indicate individual stable clones. (B) Levels of mRNAs coding for DUSP1, DUSP2, DUSP4, and DUSP5 in DUSP2-knockdown and control HeLa cells. Data show mean and SEM of 3 independent experiments. ${ }^{*} P<0.05$ compared with control. (C) Growth curves of tumors with DUSP2-knockdown (shDUSP2-1\#8 and shDUSP2-1\#12) and control (shLuciferase) cells ( $n=8$ mice/group). ${ }^{*} P<0.05$ compared with control group.

anticancer drugs. As expected, DUSP2-knockdown cells demonstrated increased resistance to paclitaxel, cisplatin, and oxaliplatin treatment in the setting of normoxia (Figure 6B and Supplemental Figure 12). Colony formation assays also demonstrated that knockdown of DUSP2 increased the number and sizes of colonies when grown in soft agar (Figure 6C). Importantly, treatment with PD98059 or U0126 to block ERK phosphorylation also abolished hypoxia-mediated drug resistance (Figure 6D and Supplemental Figure 13). Taken together, these data demonstrate that downregulation of DUSP2 in hypoxic conditions promotes drug resistance by enabling unchecked phosphorylation of ERK.

Reexpression of DUSP 2 increases drug sensitivity. Next, we employed GFP-DUSP2 HeLa cells (in which expression of the GFP-DUSP2 fusion protein is induced by doxycycline treatment) to test whether HIF-1-induced drug resistance can be abolished by reexpression of DUSP2. In the absence of doxycycline, paclitaxel-induced cell death was attenuated by hypoxia (Figure 6E and Supplemental Figure 14). In contrast, hypoxia-mediated resistance to paclitaxelinduced cell death was abolished in the presence of doxycycline. The reversal of drug resistance was not due to a nonspecific effect on doxycycline, because the addition of doxycycline under normoxia failed to affect paclitaxel-induced cell death (Figure 6E).

We then tested whether drug sensitivity could be enhanced by preventing DUSP2 downregulation during tumor growth. SCID mice ( $n=7-9$ /group) were inoculated with HeLa cells that carried the inducible DUSP2-GFP fusion gene or GFP alone and treated without or with paclitaxel (10 or $20 \mathrm{mg} / \mathrm{kg}$ body weight) in the presence or absence of doxycycline. Treatment with $20 \mathrm{mg} / \mathrm{kg}$ body weight paclitaxel delayed but did not completely inhibit tumor growth, while treatment with $10 \mathrm{mg} / \mathrm{kg}$ had no effect on growth (Figure 6F and Supplemental Figure 15). Induction of DUSP2 expression by addition of doxycycline to drinking water not only inhibited tumor growth but also caused tumor regression. The combination of DUSP2 induction and paclitaxel treatment markedly reduced tumor size (Figure 6F). The additive effect was more pronounced in the group treated with $20 \mathrm{mg} / \mathrm{kg}$ body weight paclitaxel plus doxycycline (Figure 6F and Supplemental Figure 15).

DUSP2 inversely regulates drug resistance genes. To investigate potential mechanisms for DUSP2-mediated drug resistance, we constructed a hypoxia-mediated gene regulatory network using MetaCore (Supplemental Figure 16). To test whether the expression of downstream target genes could be controlled by DUSP2, we selected one transcription factor (early growth response factor-1 [EGR-1]) and 4 effector genes (osteopontin, multidrug resistance gene-1 [MDR-1], cysteinerich angiogenic inducer [CYR61], and glucose response protein 78 [GRP78]) as readouts. mRNA expression of EGR1, an important transcription factor that increases cell survival (18-20), increased with hypoxia (Figure 7A). In parallel, promoter activity assays demonstrated that EGR1 gene activity was upregulated by hypoxia (Figure 7A). Treatment with the MEK inhibitor U0126 effectively inhibited hypoxia-induced EGR-1 expression (Figure 7B). Critically, reexpression of DUSP2 (via addition of doxycycline) significantly inhibited hypoxia-induced EGR1 expression (Figure 7C), while DUSP2 knockdown increased EGR1 expression under normoxia (Figure 7D).

Hypoxia induces expression of MDR-1 (21), CYR61 (22), GRP78 (23), and osteopontin (24), with upregulation of these genes contributing to increased drug resistance. To test whether these genes are negatively regulated by DUSP2, we performed knockdown and overexpression experiments. Under normoxia, DUSP2 knockdown significantly increased expression of MDR-1, CYR61, GRP78, and osteopontin (Figure 7E). In contrast, under hypoxic conditions DUSP2 expression significantly inhibited the expression of these genes (Figure 7F). Taken together, these data suggest that DUSP2 serves as a master regulator downstream of HIF- $1 \alpha$, regulating drug resistance and malignancy via multiple pathways. 
A

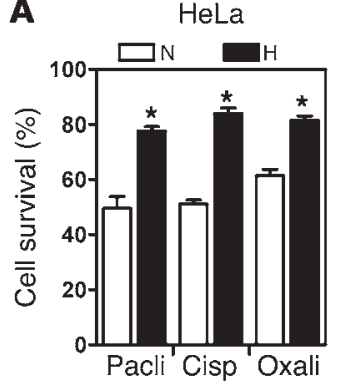

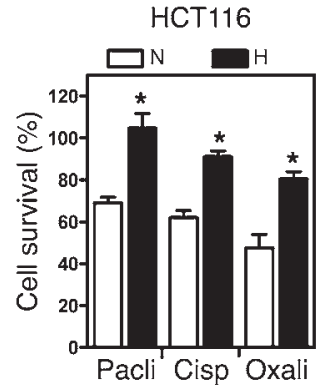

B

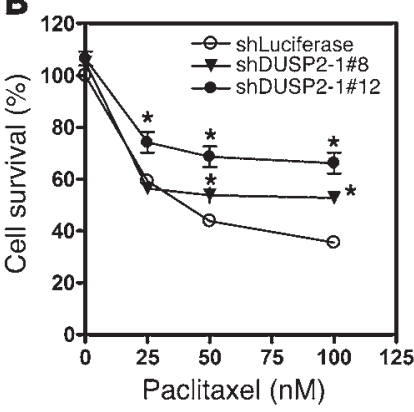

C

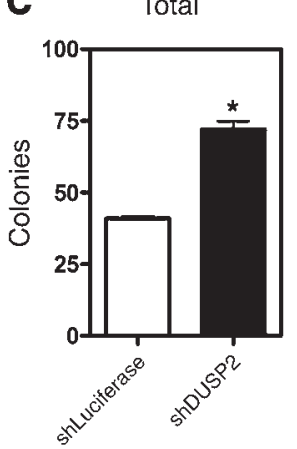

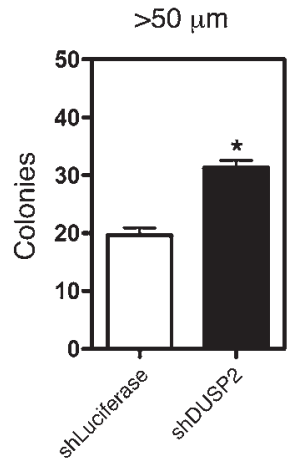

D

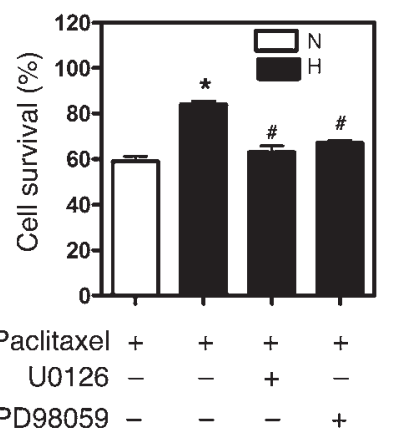

E

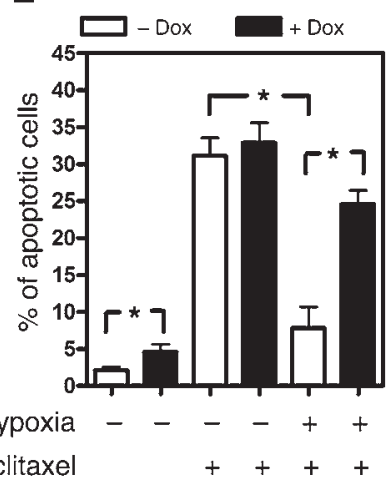

F

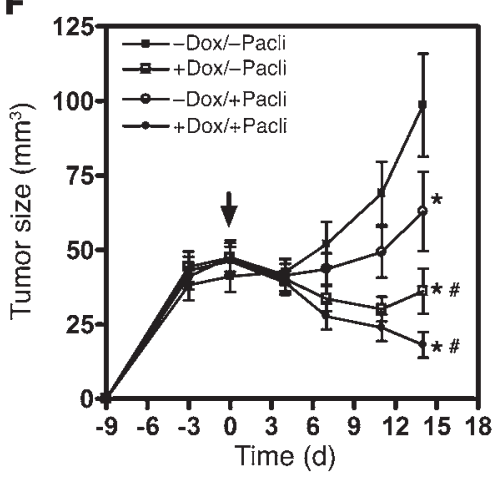

Figure 6

DUSP2 controls drug sensitivity. (A) Survival of HeLa and HCT116 cells treated with paclitaxel (Pacli, $50 \mathrm{nM})$, cisplatin (Cisp, $20 \mathrm{nM}$ ), or oxaliplatin (Oxali, $25 \mu \mathrm{g} / \mathrm{ml}$ ) under normoxia or hypoxia for 24 hours. Cell viability was determined by crystal violet staining. ${ }^{*} P<0.05$ compared with normoxia. (B) Survival of DUSP2-knockdown and control (shLuciferase) cells cultured under normoxia and treated with different doses of paclitaxel for 24 hours. ${ }^{*} P<0.05$ compared with shLuciferase group. (C) Mean and SEM of colonies grown on soft agar. DUSP2 knockdown and control (shLuciferase) cells were treated with paclitaxel and then plated on $0.6 \%$ agar for 30 days. Total number of colony and colonies with diameter greater than $50 \mu \mathrm{m}$ were determined. ${ }^{\star} P<0.05$ compared with control group. (D) Hypoxia-induced paclitaxel resistance was abolished by the inhibitors of ERK phosphorylation U0126 (10 $\mu \mathrm{M})$ and PD98059 $(50 \mu \mathrm{M})$. ${ }^{*} P<0.05$ compared with normoxia-paclitaxel group; ${ }^{\#} P<0.05$ compared with hypoxia-paclitaxel group. (E) Numbers of apoptotic HeLa cells carrying the inducible DUSP2-GFP gene cultured under normoxia or hypoxia in the presence or absence of doxycycline and treated with or without paclitaxel for 24 hours. ${ }^{*} P<0.05$. (F) Growth curves of tumors with inducible DUSP2-GFP inoculated into mice ( $n=7-9 / \mathrm{group})$ treated with or without paclitaxel (20 mg/kg body weight) combined with or without doxycycline. Arrow indicates the day treatments started. ${ }^{\star} P<0.05$ compared with control group (-Dox/-Pacli); ${ }^{\#} P<0.05$ compared with paclitaxel only group (-Dox/+Pacli).

\section{Discussion}

Despite enormous investments in cancer research, the clinical success of a multitude of therapeutic agents has been impressively poor. The most important reason for the poor performance of cancer drugs is that individual tumors consist of a heterogeneous array of transformed cells. Moreover, malignant behavior in the majority of human tumors is driven by accumulation of several genetic and epigenetic aberrations, not simply by altered activity in a single signaling pathway. This suggests that targeting a common essential pathway utilized for survival by most, if not all, cancer cells is a rational goal for the development of novel therapeutics. ERK is the convergence point of most mitogenic signaling pathways, making it an ideal target for treatment of human cancer. To date, however, no specific ERK inhibitor exists.

Aberrant activation of the ERK pathway is associated with malignancy in many cancers, including those of lung, breast, colon, ovary, bladder, and pancreas (25-27). Nonetheless, no activating mutation in ERK has been detected in human cancer (28). In addition, although activating mutations of upstream molecules such as epidermal growth factor receptor (29), Ras (30), and Raf (31) result in constitutive ERK phosphorylation, aberrant activation of ERK has also been observed in many cancers without detectable activating mutations of these genes $(25,26,32)$. These findings suggest that other unidentified mechanisms may also contribute to aberrant ERK activation. Here, we demonstrated that downregulation of DUSP2 is a critical driver of aberrant ERK activation in cancer cells.

DUSP2 is an ERK-specific phosphatase that plays important roles in innate immunity and inflammatory signaling (11). In a non-immune system, DUSP2 was found to be involved in p53-and E2F1-regulated apoptosis $(13,14)$. DUSP2 mRNA was reported to be downregulated in acute leukemia but upregulated in ovarian serous carcinoma $(33,34)$, but its role in cancer progression was unknown. In this study, we used a commercial RNA panel to determine that DUSP2 mRNA is downregulated in many human cancers. In addition, we used immunohistochemical staining to evaluate colorectal and cervical cancer samples collected at our institution. DUSP2 protein was abundant in normal colon and cervical epithelial cells but was either undetectable or markedly reduced in these two kinds of cancers, suggesting that DUSP2 

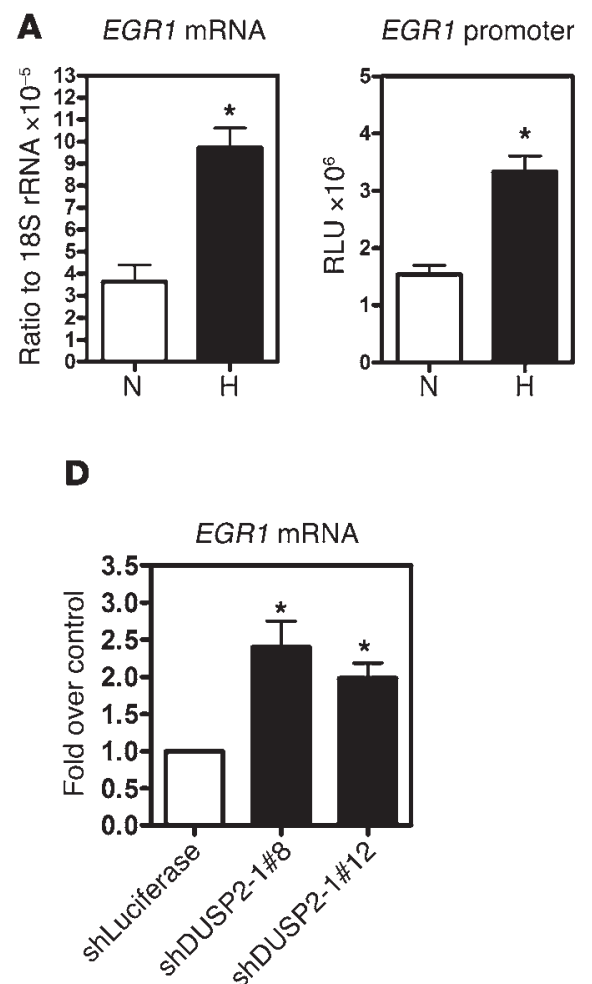

\section{B}

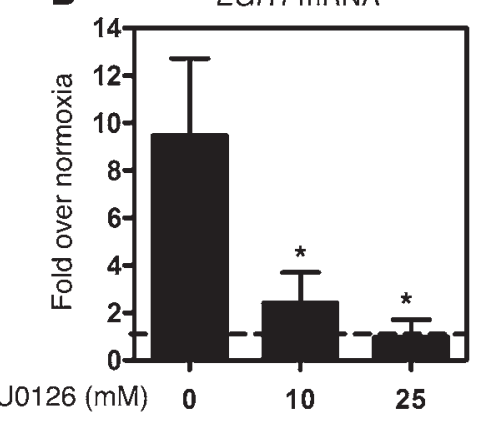

E

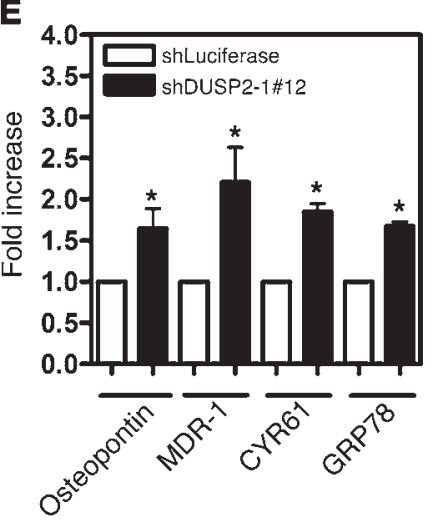

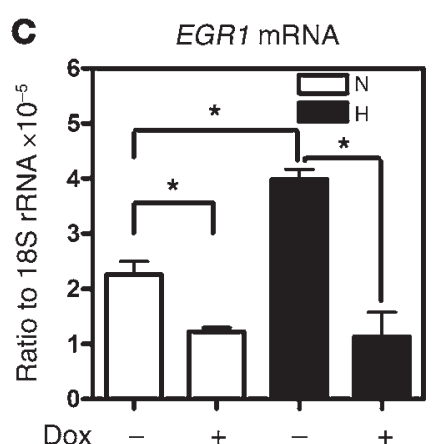

$\mathbf{F}$

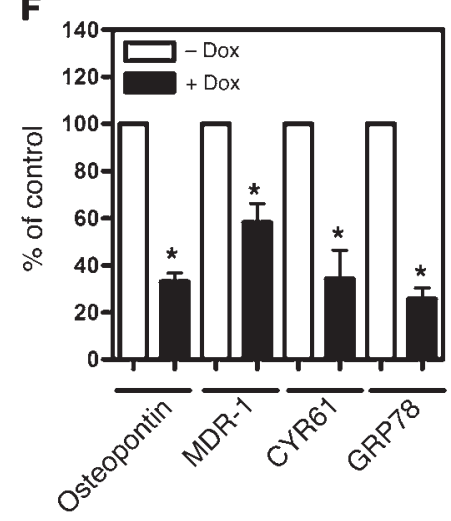

Figure 7

DUSP2 inversely controls genes involved in drug resistance. (A) Promoter activity (right panel) and mRNA levels (left panel) of EGR1 from HeLa cells cultured under normoxia or hypoxia for 24 hours. ${ }^{*} P<0.05$ compared with normoxia group. (B) Levels of EGR1 mRNA from HeLa cells cultured under normoxia or hypoxia and treated with or without U0126 for 24 hours. The level of mRNA in normoxia groups was normalized as 1 and indicated as the dash line. ${ }^{*} P<0.05$ compared with group without U0126. (C) Levels of EGR1 mRNA from HeLa cells carrying inducible DUSP2 cultured under normoxia or hypoxia and treated with or without doxycycline for 24 hours. ${ }^{*} P<0.05$. (D) Levels of $E G R 1$ mRNA from HeLa cells with (shDUSP2-1\#8 and shDUSP2-1\#12) or without (shLuciferase) DUSP2 knockdown cultured under normoxia for 24 hours. ${ }^{*} P<0.05$ compared with control group. (E) Levels of mRNA encoding for osteopontin, MDR-1, CYR61, and GRP78 from HeLa cells with (shDUSP2-1\#12) or without (shLuciferase) DUSP2 knockdown cultured under normoxia for 24 hours. ${ }^{*} P<0.05$ compared with shLuciferase group. (F) Levels of mRNA encoding for osteopontin, MDR-1, CYR61, and GRP78 from HeLa cells carrying inducible DUSP2 cultured hypoxia and treated with or without doxycycline for 24 hours. ${ }^{\star} P<0.05$ compared with without-doxycycline group.

loss of function may play critical roles in tumorigenesis. Furthermore, by examining 102 paired normal and cancerous samples, we found that levels of DUSP2 were negatively associated with malignancy in colon cancer.

Reduction of DUSP2 expression in cancer cells was an intriguing phenomenon that attracted our attention. We first established that diminished DUSP2 expression was not caused by hypermethylation of the DUSP2 promoter. Instead, DUSP2 expression was specifically inhibited by HIF- $1 \alpha$ in multiple cancer cell lines. We identified an HRE in the human DUSP2 promoter and demonstrated that HIF-1 $\alpha$ directly binds to this HRE site and inhibits DUSP2 transcription. Hypoxia-mediated DUSP2 inhibition is a unique and gene-specific phenomenon, since the expression of other nuclear DUSPs (DUSP1, -4 , and -5 ) was actually upregulated under the same conditions. Despite upregulation of DUSP1, -4 and -5 , nuclear levels of phosphorylated ERK remain high under hypoxic conditions. This phenomenon suggests that regulation of ERK phosphorylation by DUSP is a highly specific process. While 12 DUSPs have been shown to dephosphorylate ERK in a cell-free system (6), a recent study revealed that many DUSPs are in fact nonredundant regulators of ERK signaling in response to external stimuli (35). Spatial and temporal conditions, stimulation types, and crosstalk between MAPKs and DUSPs can all impact the final ERK phosphorylation outcome in living cells. Further studies are needed to dissect the interplay between nuclear DUSPs in controlling ERK phosphorylation under hypoxic conditions. Nevertheless, our results are consistent with a previous study by Laderoute et al. showing that DUSP1 was upregulated by hypoxia (36) and suggest that DUSP2 is, in fact, the key nuclear DUSP for negative regulation of ERK phosphorylation in hypoxic cells. Because HIF-1 $\alpha$ is elevated in most solid tumors, expression of DUSP2 is thus inhibited. Suppression of DUSP2 by hypoxia prolongs ERK activity, which will further enhance nuclear accumulation and transcriptional activity of HIF-1 $\alpha$ (37). This feed-forward loop may also explain the constitutive elevation of HIF- $1 \alpha$ in many solid tumors, while simultaneously explaining why DUSP2 is downregulated in a great number of different cancers.

A particularly interesting finding in this report is that inhibition of DUSP2 by hypoxia contributes substantially to chemoresistance. Paclitaxel, cisplatin, and oxaliplatin are widely used cancer chemotherapeutic drugs that exhibit clinical activity in a range of human malignancies (38). However, cancer cells, especially those in the hypoxic region of tumors, are more resistant to drug treatment, which ultimately causes chemotherapeutic 
failure (39). High ERK activity has been reported to contribute to paclitaxel resistance (40); however, the mechanism underlying ERK activation was unknown. Here, we present data demonstrating that elevated ERK activity is due to HIF- $1 \alpha$-mediated downregulation of DUSP2 expression in cancer cells. Knockdown of DUSP2 under normoxic conditions increased drug resistance, while restoration of DUSP2 in hypoxic cancer cells was sufficient to increase drug sensitivity. In vivo studies using xenografted mouse models also demonstrated that re-induction of DUSP2 expression additively increases paclitaxel sensitivity and ultimately leads to tumor regression.

Drug resistance is a major, if not the foremost, problem in cancer therapy and involves many intertwined and complex mechanisms. Many gene products and signaling pathways have been demonstrated to play some roles in enhancing drug resistance. Aberrant expression of genes with functions in drug response, angiogenesis, apoptosis, and cell survival, to name a few, often contributes notably to the acquisition of drug resistance. EGR-1, an important transcription factor that increases cell survival, is overexpressed in a majority of human cancers and contributes to tumorigenesis $(18,41,42)$. Overexpression of MDR-1 in cancer cells increases cellular drug efflux and causes resistance to multiple drugs (43). Aberrant expression of CYR-61, a secreted and matrix-associated protein involved in many cellular activities, including growth and differentiation, increases resistance to paclitaxel in breast cancer (44). GRP78, an endoplasmic reticulum chaperonic protein, is overexpressed in a panel of human breast cancer cells that have developed resistance to a variety of drug treatment regimens (23). Osteopontin, a multifunctional cytokine, enhances drug resistance by suppressing BCL-2 protein downregulation (45). These findings indicate that cancer cells utilize one or more mechanisms to acquire drug resistance. Blocking the downstream effectors of these proteins appears to be quite difficult, with outcomes likely limited because they exert distinct functions via many different signaling pathways. Here, we found that these 5 genes, despite their distinct modes of action in causing drug resistance, are negatively regulated by DUSP2. Considering that DUSP2 is downregulated by HIF-1 $\alpha$, it is not surprising that these 5 genes are upregulated under hypoxia even though no HREs were identified in some (21-24).

In conclusion, we have demonstrated that loss of function of DUSP2 not only leads to prolonged ERK activation and tumorigenesis but also contributes to hypoxia-mediated drug resistance. Because hypoxia is a common feature most solid cancers will encounter once the tumor mass exceeds $1 \mathrm{~mm}^{3}$, our findings provide insight into understanding how cancer cells become chemoresistant and reveal a mechanistic link between hypoxia and ERK activation, the two critical factors in cancer progression and malignancy. Our findings also suggest that DUSP2 may serve as a novel molecular target for cancer therapy. Further investigation into prevention of DUSP2 downregulation by hypoxia or restoration of DUSP2 under hypoxic conditions may provide a new direction for future developments in cancer therapy.

\section{Methods}

Cell culture and treatment. Cancer cells were cultured in medium containing $10 \% \mathrm{FBS}$ and antibiotics $(100 \mu \mathrm{g} / \mathrm{ml}$ streptomycin and $100 \mathrm{U} / \mathrm{ml}$ penicillin G) in a humidified atmosphere of $5 \% \mathrm{CO}_{2}$ and $95 \%$ air at $37^{\circ} \mathrm{C}$. SW 480 cells were cultured in the absence of $\mathrm{CO}_{2}$ as previously described (46). When cells reached $70 \%$ confluence, cultured medium was changed to serum-free medium for 16 hours. After starvation and the addition of fresh medium containing $10 \%$ FBS, cells were treated with true hypoxia $\left(1 \% \mathrm{O}_{2}, 5 \% \mathrm{CO}_{2}\right.$, and $\left.94 \% \mathrm{~N}_{2}\right)$ or chemical hypoxia (DFO or DMOG) for the indicated times.

RT-qPCR and Western blotting. Total RNA (50 ng) was subjected to RT-qPCR as previously described (47). Transcripts were quantified by ABI 7900 thermocycler (Applied Biosystems). Each reaction contained $50 \mathrm{ng}$ of RT products, $0.3 \mu \mathrm{M}$ of specific primers (Supplemental Table 2), and $10 \mu \mathrm{l}$ of SYBR Green mix.

The proteins were extracted by TRIzol reagent according to the manufacturer's instructions (Invitrogen) and dissolved in 1\% SDS buffer containing $5 \mathrm{mM}$ DTT, $1 \mathrm{mM}$ PMSF, $10 \mu \mathrm{g} / \mathrm{ml}$ aprotinin, $10 \mu \mathrm{g} / \mathrm{ml}$ pepstatin A, $50 \mu \mathrm{g} / \mathrm{ml}$ leupeptin, $2.5 \mathrm{mM} \mathrm{Na}$-pyrophosphate, and $1 \mathrm{mM} \mathrm{Na} \mathrm{VO}_{4}$. An equal amount of protein was loaded into each lane, separated on an SDS-PAGE gel, and transferred to a polyvinylidene difluoride membrane. The membrane was blocked in 5\% nonfat milk at room temperature for 1 hour and then incubated in primary antibody (DUSP2, 1:200; HIF-1 $\alpha$, 1:1,000; HIF- $2 \alpha, 1: 2,500$; HIF- $1 \beta, 1: 1,000)$ at $4{ }^{\circ} \mathrm{C}$ overnight. The DUSP2 antibody was purchased from Santa Cruz Biotechnology Inc. (catalog sc-32776); antibodies for HIF proteins were described previously (16). After incubation with horseradish peroxidase-conjugated secondary antibody $(1: 5,000)$ and washing, signals were detected using an enhanced chemiluminescence detection kit (PerkinElmer). The membrane was stripped with stripping buffer at $55^{\circ} \mathrm{C}$ for 30 minutes and redetected using a second antibody as described above.

Identification of HRE and promoter activity assays. Human DUSP2 promoter sequence was analyzed using an in-house bioinformatic platform, The BEST (http://www.binfo.ncku.edu.tw/thebest/), to predict HIF-1-binding sites. Sequence homology across several mammalian species was analyzed by multiple sequence alignment. The fragment of the human DUSP2 5' flanking region (-998 to $+77 \mathrm{bp}$ ) was cloned into pGL3-basic vector containing the luciferase reporter system. Site-directed mutagenesis of putative HRE constructs was generated using PCR amplification. A commercial plasmid containing a CMV-driven $\beta$-gal reporter system was used as an internal control. Cells were plated in 24-well plates for the luciferase/ $\beta$-gal assays. Plasmids were transfected using Lipofectamine 2000 (Invitrogen). Transfection was followed by rinsing and incubation in 10\% DMEM/F12 medium for 6 hours. After the medium was changed, cells were allowed to recover for 16 hours and then exposed to hypoxic conditions in $10 \%$ DMEM/F12 for 24 hours. Luciferase assays were performed using the DualLuciferase Reporter Assay System according to the manufacturer's instructions (Promega). Briefly, $50 \mu \mathrm{l}$ luciferase substrate was added to $20 \mu \mathrm{l}$ lysate, and luciferase activity was measured using a 20/20 Luminometer (Turner Designs). Each luciferase assay was performed in triplicate.

Construction of expression plasmids. To generate a wild-type DUSP2 expression vector, we designed primers containing XhoI and HindIII from the full-length DUSP2 mRNA sequence. Amplified DUSP2 cDNA was cloned into PEGFP-N1 vector. The inducible DUSP2-GFP vector was generated by using EcoRV and NotI to digest DUSP2-GFP from pEGFP-N1 vector and then subcloned into pcDNA5/To vector (Invitrogen). T-REx-HeLa cells (from Invitrogen) were transfected with the inducible DUSP2-GFP vector. After selection by blasticidin $\mathrm{S}$ and hygromycin B for 2 months, stable clones of inducible DUSP2-GFP were established.

Transient and stable RNA knockdown. siRNAs against HIF- $1 \alpha$ and HIF- $2 \alpha$ and scrambled siRNA control (Supplemental Table 3) from Invitrogen were used for transient knockdown assays. A final concentration of $40 \mathrm{nM}$ siRNA was transfected by Lipofectamine 2000 and incubated for 6 hours. After incubation, HeLa cells were directly cultured under hypoxia or normoxia for 24 hours. To generate stable clones with DUSP2 knockdown, we used a bioinformatic Web tool to design shRNAs against DUSP2 (shRNA Explorer from GeneLink), and two different target sites were selected to synthesize 
oligonucleotides (Supplemental Table 3). According to the manufacturer's procedure, shDUSP2 oligonucleotides were cloned into pSingle-tTs-shRNA vector (Clontech). HeLa cells were transfected with shDUSP2 plasmids by Lipofectamine 2000. After selection by $500 \mu \mathrm{g} / \mathrm{ml} \mathrm{G} 418$ (Sigma-Aldrich) for 2 months, several stable knockdown clones were established and verified.

ChIP assay. Protein (HIF-1 $\alpha$ ) and DNA from cells exposed to normoxia or hypoxia $\left(1 \times 10^{6}\right.$ cells) were cross-linked by incubation for 10 minutes at $37^{\circ} \mathrm{C}$ with a final concentration of $1 \%$ formaldehyde. The ChIP assay was performed as previously described (16). DNA was released from the protein-DNA complex and subjected to 35 cycles of PCR amplification using the primers listed in Supplemental Table 2.

TUNEL assay. Apoptotic cells were detected by using a commercial detection kit (TdT-FragEL DNA Fragmentation Detection Kit, Calbiochem) according to the manufacturer's procedure. For each experiment, at least 1,300 cells were counted to determine the number of apoptotic nuclei.

Collection of cancer and normal tissues and immunohistochemistry. The mRNA panel of normal and cancer tissues was purchased from OriGene. For each cancer type, it contains 3 normal tissue and $4-8$ cancerous samples. For immunohistochemistry, tumor specimens were obtained from 18 patients with cervical cancer and 102 patients with colon cancer who underwent surgery at the Department of Surgery of National Cheng Kung University Hospital. The survival information was not available because the time for follow-up was not long enough (less than 4 years). Specimens of tumor tissue and adjacent tissues were collected. The stage of each tumor was classified and histologically confirmed by pathologists. Ethics approval for human studies was obtained from the Clinical Research Ethics Committee at National Cheng Kung University Medical Center, and informed consent was given by each patient. The procedure used for immunohistochemical staining was described previously (46).

Xenograft mouse model. Inducible GFP or DUSP2-GFP cells $\left(5 \times 10^{6}\right)$ suspended in $100 \mu \mathrm{l}$ of $1 \times$ PBS were inoculated s.c. in the hind flank of male SCID mice (6-8 weeks old, from the Animal Center, College of Medicine, National Cheng Kung University). The mice were housed in barrier facilities on a 12-hour light/12-hour dark cycle with food and water available ad libitum. All procedures were performed under protocols approved by the Institutional Animal Care and Use Committee of the National Cheng Kung University. After the tumors reached about $50 \mathrm{~mm}^{3}$, the mice were randomly assigned into experimental or control groups. Mice in the experimental group were given drinking water containing $2 \mu \mathrm{g} / \mathrm{ml}$ doxycycline, while mice in the control group received normal drinking water. Paclitaxel $(10$ or $20 \mathrm{mg} / \mathrm{kg}$ body weight) or saline was given by intraperitoneal injection on days 3 and 6 of a 7 -day cycle for 2 cycles. Tumors were measured with calipers every 3-4 days during the experimental period. Tumor volumes were calculated by the following formula: $a \times b \times c \times 0.52$, where $a$ is the length, $b$ is the width, and $c$ is the height of the tumor. After mice were sacrificed, tumor weight was recorded. Tumors were dissected, fixed in $4 \%$ paraformaldehyde, and embedded in paraffin for immunohistochemical staining.

Confocal microscopy. HeLa cells were transiently transfected with GFP or DUSP2-GFP for 24 hours and then fixed in 3.7\% formaldehyde. After washing, cells were treated with $0.5 \%$ Triton $\mathrm{X}-100$ and then washed again in $1 \times$ PBS. After blocking by Superblock buffer (Thermo Scientific), cells were incubated with phospho-ERK1/2 antibody (1:200) (Cell Signaling Technology) and Hoechst $(1: 1,000)$ at $4^{\circ} \mathrm{C}$ overnight. After washing in $1 \times$
PBST, cells were incubated with secondary antibody (Alexa Fluor 594-goat anti-rabbit; Invitrogen) for 1 hour at room temperature and then washed again in $1 \times$ PBST. Finally, the cells were observed on a scanning confocal laser microscope (Leica TCS SPII).

Cell viability assay. Cells were treated with different doses of paclitaxel, cisplatin, or oxaliplatin and cultured under hypoxia or normoxia for 24 or 48 hours. After incubation, the detached cells were removed by washing 3 times with serum-free medium, while attached cells were fixed with $1 \%$ glutaraldehyde for 10 minutes and stained with $0.1 \%$ crystal violet (Fisher Scientific) for 25 minutes. The plate were re-immersed in fresh tap water to remove excess dye and then allowed to air dry for 5-10 minutes. A total of $50 \mu \mathrm{l}$ of $0.5 \%$ Triton X-100 was added to each well to solubilize the cells overnight at room temperature. The absorbance was measured at $\mathrm{OD}_{595}$ using a microplate reader.

Colony formation assay. The stable DUSP2 or luciferase knockdown clones were treated with paclitaxel $(50 \mathrm{nM})$, and then suspended in $0.3 \%$ agar with complete DMEM/F12 medium. Cells were plated at a density of 5,000 cells per $30-\mathrm{mm}$ dish precoated with $0.6 \%$ agar and maintained at $37^{\circ} \mathrm{C}$. On day 30 , the colonies were stained by $0.1 \%$ crystal violet, and numbers of colonies were determined under a microscope at $\times 40$ magnification. Each stable clone had 6 plates, and a total of 10 fields were counted for each plate.

Gene regulatory network. MetaCore (GeneGo) installed in the Bioinformatics Center of National Cheng Kung University was used to build the gene regulatory network. The inhibitory relationship of HIF-1 $\alpha$ and DUSP2 was manually added based on our current findings. The rest of the gene regulatory relationships were automatically built by the software using the following criteria: (a) genes with transcription function directly interacting with ERK were selected; (b) 4 major transcription factors (EGR-1, Elk-1, c-Myc, and c-Fos) were selected to construct the second layer of network; and (c) only genes involved in drug response, angiogenesis, cell survival, and apoptosis according to the gene ontology description were selected to build the network downstream of the 4 transcription factors.

Statistics. The data are expressed as mean \pm SEM. Two-tailed Student's $t$ test was used to compare differences between 2 groups. Differences between groups were analyzed using 1-way and 2-way ANOVA, followed by post-test analysis using commercial statistical software (GraphPad Prism 4.02, GraphPad Software). Statistical significance was set at $P<0.05$.

\section{Acknowledgments}

We thank the Bioinformatics Center of National Cheng Kung University for providing bioinformatic analytic tools (The BEST and MetaCore). We also thank Eric D. Buras for critical reading and editing of the manuscript. This work was supported by grants from the National Health Research Institute (NHRI-EX9932BI) and the National Research Program for Genomic Medicine (NSC98-3112B-006-010), Taiwan.

Received for publication July 14, 2010, and accepted in revised form January 26, 2011.

Address correspondence to: Shaw-Jenq Tsai, Department of Physiology, College of Medicine, National Cheng Kung University, Tainan 701, Taiwan. Phone: 886.6.2353535, ext. 5426; Fax: 886.6.2362780; E-mail: seantsai@mail.ncku.edu.tw.
1. Bruick RK, McKnight SL. A conserved family of prolyl-4-hydroxylases that modify HIF. Science. 2001;294(5545):1337-1340.

2. Maxwell PH, et al. The tumour suppressor protein VHL targets hypoxia-inducible factors for oxygen-dependent proteolysis. Nature. 1999;399(6733):271-275.

3. Jaakkola P, et al. Targeting of HIF-alpha to the von Hippel-Lindau ubiquitylation complex by $\mathrm{O} 2$-regulated prolyl hydroxylation. Science. 2001;292(5516):468-472.

4. Semenza GL. Defining the role of hypoxia-inducible factor 1 in cancer biology and therapeutics. Oncogene. 2010;29(5):625-634.

5. Brown JM, Wilson WR. Exploiting tumour hypoxia in cancer treatment. Nat Rev Cancer.
2004;4(6):437-447.

6. Owens DM, Keyse SM. Differential regulation of MAP kinase signalling by dual-specificity protein phosphatases. Oncogene. 2007;26(22):3203-3213.

7. Alonso A, et al. Protein tyrosine phosphatases in the human genome. Cell. 2004;117(6):699-711.

8. Theodosiou A, Ashworth A. MAP kinase phosphatases. Genome Biol. 2002;3(7):REVIEWS3009. 
9. Rohan PJ, et al. PAC-1: a mitogen-induced nuclear protein tyrosine phosphatase. Science. 1993; 259(5102):1763-1766.

10. Zhang Q, Muller M, Chen CH, Zeng L, Farooq A, Zhou MM. New insights into the catalytic activation of the MAPK phosphatase PAC-1 induced by its substrate MAPK ERK2 binding. J Mol Biol. 2005;354(4):777-788

11. Lang R, Hammer M, Mages J. DUSP meet immunology: dual specificity MAPK phosphatases in control of the inflammatory response. J Immunol. 2006;177(11):7497-7504.

12. Jeffrey KL, et al. Positive regulation of immune cell function and inflammatory responses by phosphatase PAC-1. Nat Immunol. 2006;7(3):274-283.

13. Wu J, Jin YJ, Calaf GM, Huang WL, Yin Y. PAC1 is a direct transcription target of E2F-1 in apoptotic signaling. Oncogene. 2007;26(45):6526-6535.

14. Yin Y, Liu YX, Jin YJ, Hall EJ, Barrett JC. PAC1 phosphatase is a transcription target of p53 in signalling apoptosis and growth suppression. Nature. 2003;422(6931):527-531.

15. Kim JW, Tchernyshyov I, Semenza GL, Dang CV. HIF1-mediated expression of pyruvate dehydrogenase kinase: a metabolic switch required for cellular adaptation to hypoxia. Cell Metab. 2006;3(3):177-185.

16. Lu CW, Lin SC, Chen KF, Lai YY, Tsai SJ. Induction of pyruvate dehydrogenase kinase- 3 by hypoxia-inducible factor- 1 promotes metabolic switch and drug resistance. J Biol Chem. 2008;283(42):28106-28114.

17. Papandreou I, Cairns RA, Fontana L, Lim AL, Denko NC. HIF-1 mediates adaptation to hypoxia by actively downregulating mitochondrial oxygen consumption. Cell Metab. 2006;3(3):187-197.

18. Abdulkadir SA, et al. Impaired prostate tumorigenesis in Egr1-deficient mice. Nat Med. 2001;7(1):101-107.

19. Kaufmann K, Thiel G. Epidermal growth factor and platelet-derived growth factor induce expression of Egr-1, a zinc finger transcription factor, in human malignant glioma cells. J Neurol Sci. 2001; 189(1-2):83-91.

20. Mayer SI, Rossler OG, Endo T, Charnay P, Thiel G. Epidermal-growth-factor-induced proliferation of astrocytes requires Egr transcription factors. $J$ Cell Sci. 2009;122(Pt 18):3340-3350.

21. Comerford KM, Wallace TJ, Karhausen J, Louis NA, Montalto MC, Colgan SP. Hypoxia-inducible factor1-dependent regulation of the multidrug resistance (MDR1) gene. Cancer Res. 2002;62(12):3387-3394.

22. Lin MT, et al. Involvement of hypoxia-inducing factor-1alpha-dependent plasminogen activa- tor inhibitor-1 up-regulation in Cyr61/CCN1induced gastric cancer cell invasion. J Biol Chem. 2008;283(23):15807-15815.

23. Dong D, et al. Vascular targeting and antiangiogenesis agents induce drug resistance effector GRP78 within the tumor microenvironment. Cancer Res. 2005;65(13):5785-5791.

24. Zhu Y, et al. Hypoxia upregulates osteopontin expression in NIH-3T3 cells via a Ras-activated enhancer. Oncogene. 2005;24(43):6555-6563.

25. Gioeli D, Mandell JW, Petroni GR, Frierson HF Jr, Weber MJ. Activation of mitogen-activated protein kinase associated with prostate cancer progression. Cancer Res. 1999;59(2):279-284.

26. Hoshino $\mathrm{R}$, et al. Constitutive activation of the 41-/43-kDa mitogen-activated protein kinase signaling pathway in human tumors. Oncogene. 1999 18(3):813-822.

27. Sebolt-Leopold JS, et al. Blockade of the MAP kinase pathway suppresses growth of colon tumors in vivo. Nat Med. 1999;5(7):810-816

28. Kohno M, Pouyssegur J. Targeting the ERK signaling pathway in cancer therapy. Ann Med. 2006; 38(3):200-211.

29. Hayman MJ, Enrietto PJ. Cell transformation by the epidermal growth factor receptor and v-erbB. Cancer Cells. 1991;3(8):302-307.

30. Bos JL. ras oncogenes in human cancer: a review. Cancer Res. 1989;49(17):4682-4689.

31. Wan PT, et al. Mechanism of activation of the RAFERK signaling pathway by oncogenic mutations of B-RAF. Cell. 2004;116(6):855-867.

32. Oka H, et al. Constitutive activation of mitogenactivated protein (MAP) kinases in human renal cell carcinoma. Cancer Res. 1995;55(18):4182-4187.

33. Givant-Horwitz V, Davidson B, Goderstad JM, Nesland JM, Trope CG, Reich R. The PAC-1 dual specificity phosphatase predicts poor outcome in serous ovarian carcinoma. Gynecol Oncol. 2004, 93(2):517-523.

34. Kim SC, Hahn JS, Min YH, Yoo NC, Ko YW, Lee WJ. Constitutive activation of extracellular signal-regulated kinase in human acute leukemias: combined role of activation of MEK, hyperexpression of extracellular signal-regulated kinase, and downregulation of a phosphatase, PAC1. Blood. 1999;93(11):3893-3899.

35. Caunt CJ, Armstrong SP, Rivers CA, Norman MR, McArdle CA. Spatiotemporal regulation of ERK2 by dual specificity phosphatases. J Biol Chem. 2008; 283(39):26612-26623.
36. Laderoute KR, Mendonca HL, Calaoagan JM, Knapp AM, Giaccia AJ, Stork PJ. Mitogen-activated protein kinase phosphatase-1 (MKP-1) expression is induced by low oxygen conditions found in solid tumor microenvironments. A candidate MKP for the inactivation of hypoxia-inducible stress-activated protein kinase/c-Jun $\mathrm{N}$-terminal protein kinase activity. J Biol Chem. 1999;274(18):12890-12897.

37. Richard DE, Berra E, Gothie E, Roux D, Pouyssegur J. p42/p44 mitogen-activated protein kinases phosphorylate hypoxia-inducible factor 1alpha (HIF1alpha) and enhance the transcriptional activity of HIF-1. J Biol Chem. 1999;274(46):32631-32637.

38. Arbuck SG, et al. Clinical development of Taxol. J Natl Cancer Inst Monogr. 1993;(15):11-24.

39. McDaid HM, Horwitz SB. Selective potentiation of paclitaxel (taxol)-induced cell death by mitogen-activated protein kinase kinase inhibition in human cancer cell lines. Mol Pharmacol. 2001; 60(2):290-301.

40. MacKeigan JP, Collins TS, Ting JP. MEK inhibition enhances paclitaxel-induced tumor apoptosis. J Biol Chem. 2000;275(50):38953-38956.

41. Ma J, et al. Targeted knockdown of EGR-1 inhibits IL-8 production and IL-8-mediated invasion of prostate cancer cells through suppressing EGR-1/NF-kappaB synergy. J Biol Chem. 2009; 284(50):34600-34606

42. Mahalingam D, Natoni A, Keane M, Samali A, Szegezdi E. Early growth response-1 is a regulator of DR5-induced apoptosis in colon cancer cells. BrJ Cancer. 2010;102(4):754-764.

43. Gottesman MM, Ling V. The molecular basis of multidrug resistance in cancer: the early years of P-glycoprotein research. FEBS Lett. 2006; 580(4):998-1009.

44. Lin MT, et al. Cyr61 expression confers resistance to apoptosis in breast cancer MCF-7 cells by a mechanism of NF-kappaB-dependent XIAP up-regulation. J Biol Chem. 2004;279(23):24015-24023.

45. Gu T, et al. Osteopontin is involved in the development of acquired chemo-resistance of cisplatin in small cell lung cancer. Lung Cancer. 2009; 66(2):176-183

46. Chien C-W, et al. Regulation of CD151 by hypoxia controls cell adhesion and metastasis in colorectal cancer. Clin Cancer Res. 2008;14(24):8043-8051.

47. Chuang PC, Wu MH, Shoji Y, Tsai SJ. Downregulation of CD36 results in reduced phagocytic ability of peritoneal macrophages of women with endometriosis. J Pathol. 2009;219(2):232-241. 